\title{
3. Literaturverzeichnis
}

Amelunxen

Entschädigung der Opfer strafbarer Handlungen;

hier: Wiedergutmachung im Strafprozeß, RIDP.

1973 S. 19

ders.,

Die Entschädigung des durch die Straftat Verletzten, ZStW 1974 (Bd. 86) S. $457 \mathrm{f}$.

ders.,

Das Opfer der Straftat, 1970

Atiyah

Accidents, Compensation and the Law, London 1970

Baldus, Willms, Hsg.

Leipziger Kommentar, Strafgesetzbuch, 9. Aufl.

Bockelmann

Strafrecht, Allgemeiner Teil, 2. Aufl, München 1975

Brackmann

Handbuch der Sozialversicherung

Asgard-Verlag, Bad Godesberg

Breithaupt

Sammlung von Entscheidungen der Sozialversicherung, Versorgung und Arbeitslosenver- 
Literaturverzeichnis

sicherung

Walter Stutz Verlag, München

Brooks

Compensating Victims of Crime: The Recommendations of Programm Administrators, Law \&

Society Review (LSR) 1973, Vol. 7, Nr. 3

Dersch-Knoll-Brockhoff u. a.

RVO-Gesamtkommentar

Verlag Chmielorz, Wiesbaden

Drapkin, Viano

Victimology: A New Focus, Vol. I-International Symposium on Victimology, Jerusalem 1973

Dreher

Strafgesetzbuch, Kommentar, 36. Aufl. 1976

Edelhertz, Geis

Public Compensation to Victims of Crime,

Praeger Publishers, NY., 1974

Eichler

Versicherungsrecht, Karlsruhe 1966

Eilsberger

Die Kölner Straßenbahnblockade, JuS 1970, 164

Entwurf eines Gesetzes über die Entschädigung für Opfer von Gewalttaten - der Bundesregierung vom 27. 8. 1974 - BT.-Drucks. 7/2506;

dazu: Anlage 2, Stellungnahme des Bundesrates S. 19; 
Anlage 3, Gegenäußerung der Bundesregierung

S. 23

Entwurf eines Gesetzes über Hilfe für Opfer von Straftaten,

eingebracht von der Fraktion der CDU/CSU im BT., Drucks. VI/2420.

Beratung in der 134. Sitzung des BT. am 22. 9. 1971, Prot. S. 7812

Flister

Das englische Criminal Injuries Compensation Scheme, NJW $70,985 \mathrm{f}$

\section{Forsthoff}

Lehrbuch des Verwaltungsrechts

C.H. Beck-Verlag

Geilen

Neue Entwicklungen beim strafrechtlichen Gewaltbegriff, Festschrift für Hellmuth Mayer, Berlin 1966 S. $445 f$

Göppinger

Kriminilogie, 2. Aufl., München 1973

Grebing

Die Möglichkeiten der Entschädigung des Opfers einer Straftat im französischen Recht, Revue Internationale de Droit Pénal, 44. Année (RIDP), 1973 S. $338 \mathrm{f}$

Groeben, von der; v. Boeckh, Thiesing Kommentar zum EWG-Vertrag, 2 Bde., 2. Auflage, Baden-Baden 1974 
Hastler

Aufbau und Verfahren der Sozialgerichtsbarkeit

- Kommentar zum SGG -

Hauck-Haines

Sozialgesetzbuch - Allgemeiner Teil

Kommentar

Erich Schmidt Verlag, Berlin-Bielefeld-München

v. Hentig

Das Verbrechen, Band II, 1962 (Berlin, Göttingen, Heidelberg)

v. Hippel

Staatliche Entschädigung für Verbrechensopfer, ZRP 1971, 5

Huber

Die Entschädigung des Verletzten - Iösungen

im Common-Law-Bereich, RIDP. $1973 \mathrm{~s} .321 \mathrm{f}$

\section{International Conference (3.)}

on Compensation for Victims of Crime in Toronto/Kan. - Background Material, zitiert: 3 . ICT.

Ipsen

Europäisches Gemeinschaftsrecht, Tübingen 1972

Jacob

Reparation or Restitution by the Criminal

Offender to his Victim: Applicability of an ancient Concept in the modern Correctional

Process,

The Journal of Criminal Law, Criminology and

Police Science (JCL), 1970 - Northwestern 
University School of Law, Vol. 61 No. 2 S. $152 \mathrm{ff}$

$\underline{\text { Jahn }}$

Sozialgesetzbuch (SGB) für die Praxis

Kommentar

Rudolf Haufe Verlag, Freiburg

Jantz-Zweng

Das neue Recht der Rentenversicherung der Arbeiter und Angestellten (Loseblattausgabe)

Verlag W. Kohlhammer, Stuttgart

Jescheck

Lehrbuch des Strafrechts, Allgemeiner Teil,

2. Aufl. 1972

Jung-Cramer

Das neue Schwerbehindertengesetz

Verlag Franz Vahlen, München

Kaiser

Kriminologie, 2. Auflage, Karlsruhe 1973

Knodel

Der Begriff der Gewalt im Strafrecht, München, Berlin 1962

Kommentar zum Bonner Grundgesetz

(versch. Bearbeiter)

Hanseatischer Gildenverlag

Krämer

Verbesserung des Systems der Entschädigung von Unfallopfern in England, VersR 1967, $12 \mathrm{ff}$ 
Literaturverzeichnis

\section{Irekner}

Strafgesetzbuch - Kommentar, 10. Auflage 1976

Leuterbach

Gesetzliche Unfallversicherung Kommentar

W. Kohlhammer, Stuttgart, Berlin, Köln, Mainz

\section{Lobedanz}

Schadensausgleich bei Straftaten in Spanien und Lateinamerika, Frankfurt/M. 1972

Materialien - Auswahl

BRat: Empfehlungen der Ausschüsse v. 7. 6. 1974, BR.-Drucks. 352/1/74

BR.-Stellungn. v. 21. 6. 1974, 407. Sitzung, Prot. S. 271 ff nebst Anlagen $10-12$

Beratung in der 431. Sitzung des BR. am 20. 2. 1976 (Anmufung des Vermittlungsausschusses), Prot. S. 43 ff

Unterrichtung durch den BR.

hier: Anrufung des Vermittlungsausschusses v. 24. 2. 1976, BT.-Drucks. 7/4804

Beschluß v. 9. 4. 1976: Zustimmung des BR., Drucks. 220/76

BT.: 1. Beratung - 123. Sitzg. v. 11. 10. 1974, Sten. Prot. S. $8240 f$

Bericht u. Antrag des RA. v. 21. 1. 1976, BT.-Drucks. 7/4614,

Bericht des Haushalts-A. v. 22. 1. 1976, BT.-Drucks. 7/4615,

2. und 3. Beratung in d. 219. Sitzung v. 30. 1. 1976, Sten. Prot. S. 15240 D f. 
Vermittlungsausschuß

Antrag v. 22. 3. 1976; BT-Drucks. 7/4907.

BT.-Plenum, 233. Sitzg. v. 1. 4.1976, Zustimmung zu dem Antrag des Vermittlungsausschusses, S. 16226.

Maunz-Dürig-Herzog

Grundgesetz

- Kommentar -

C.H. Beck, München

Maurach

Deutsches Strafrecht, Allgemeiner Teil,

4. Aufl. 1971, Karlsruhe

Müller

Straßenverkehrsrecht, 22. Aufl., Berlin 1969, Bd. II S. 234 ff: Pfivg

Neubert-Becke

Schwerbehindertengesetz

Kommentar

Walter Stutz Verlag, München

Niemeyer

Genugtuung des Verletzten durch Buße,

Eine Untersuchung der Bußvorschriften im

Alternativ-Entwurf, Tübingen 1972 
Literaturverzeichnis

Olshausen

Strafgesetzbuch - Kommentar, 12. Aufl. 1942

Palandt

Bürgerliches Gesetzbuch, Kommentar, 35. Aufl., 1976

Peters, Hans

Lehrbuch der Verwaltung (1949)

Springer-Verlag, Berlin-Göttingen

Peters, Horst

Allgemeiner Teil des Sozialgesetzbuchs

- Kommentar - (Loseblattausgabe)

Verlag W. Kohlhammer, Stuttgart

\section{Peters-Hommel}

Sozialgesetzbuch - Allgemeiner Teil

Textausgabe mit ergänzenden Rechtsvorschriften, einer erläuternden Einführung, Anmerkungen und Verweisungen

Verlag W. Kohlhammer, Stuttgart

\section{Peters-Sautter-Wolff}

Sozialgerichtsgesetz

Kommentar

Kohlhammer Verlag, Stuttgart

Piduch

Bundeshaushaltsrecht (Loseblattausgabe)

Kohlhammer, Stuttgart, Berlin, Köln, Mainz

Preisendanz

Strafgesetzbuch, Kommentar (ehem. Petters)

29. Aufl. 1975

$\mathrm{XX}$ 


\section{Prölss-Martin}

VersicherungsvertragsG, 20. Aufl. 1975

Pflichtversicherungs im Anhang, S. 1120

Vo. über den Entschädigungsfonds vom 14. 12.

1965 im Anhang S. 1129

7. jährlicher Report des Crime Victims Compensation Board

Legislative Document (1974) No 94 des State of New York

RGRK

Das Bürgerliche Gesetzbuch, Kommentar, herausgegeben von Mitgliedern des BGH, 12. Aufl. 1975

Roeckner-Bluschke

Kommentar zum Bundesversorgungsgesetz

Asgard-Verlag, Bad Godesberg

Roemer

Die versorgungsrechtliche Kausaltheorie im Spiegel einer allgemeinen Haftungslehre, SGb 13/63 S. 392

\section{Rohr-Beuster-Sträßer}

Bundesversorgungsrecht mit Verfahrensrecht - Handkommentar -

Verlag Dr. Pelzer, Düsseldorf

Rudolphi u. a.

Systematischer Kommentar zum StGB, Bd. 1, Frankfurt am Main 1975 
Rüfner

Empfiehlt es sich, die soziale Sicherung für den Fall von Personenschäden, für welche die Allgemeinheit eine gesteigerte Verantwortung trägt, neu zu regeln?

Gutachten E zum 49. Deutschen Juristentag 1972 in Düsseldorf

ders.,

Die Entschädigung für Opfer von Gewalttaten, NJW 1976, 1249

\section{Sabel}

Sozialgesetzbuch (SGB)

Textausgabe mit Begründung, Anmerkungen und weiterfuhrenden Hinweisen

Asgard-Verlag, Bonn-Bad Godesberg

Sauerwein

Gesetz über das Verwaltungsverfahren der Kriegsopferversorgung und seine Anwendung Kommentar (Loseblattausgabe)

Fachverlag CW Haarfeld, Essen

Schafer

The Victim and his Criminal, insbes. S. $39 \mathrm{f.:}$ Criminal - Victim Relationship as a Crime Factor, New York 1968

Schätzler

Compensation for Victims of Criminal Acts, RIDP. S. 29 f

ders.,

Versorgung für die Opfer von Gewalttaten, Der Versorgungsbeamte 1976 S. $65 \mathrm{f}$ 
Schieckel-Gurgel

Bundesversorgungsgesetz
Kommentar (Loseblattausgabe)
R. Schulz-Verlag, München

$\underline{\text { Schmidhäuser }}$

Strafrecht, AT, Lehrbuch, 2. Aufl. Tübingen 1975

Schmidt-Bleibtreu Klein

Kommentar zum Grundgesetz - 2. Auflage Iuchterhand Verlag

Schönke-Schröder

Strafgesetzbuch, Kommentar, 18. Aufl. 1976

Schönleiter

Bundesversorgungsgesetz

Kommentar, 1953

Kohlhammer Verlag, Stuttgart

ders.,

Handbuch der Bundesversorgung

Verlag Vahlen, München

Schönleiter-Hennig

Gesetz über das Verwaltungsverfahren der Kriegsopferversorgung

Kommentar

Verlag Vahlen, Berlin-Frankfurt

Schoreit

Entschädigung der Verbrechensopfer als öffentliche Aufgabe, Berlin 1973 (zitiert: Schoreit, Entschädigung) 
Literaturverzeichnis

ders.,

Multilaterale Regelung der Entschädigung für Opfer von Straftaten, ZRP 1974, 55

ders.,

Entschädigung für Opfer von Gewalttaten, Recht und Gesellschaft (RuG) 1974, $115 \mathrm{f}$

ders.,

Entschädigung für Verbrechensopfer, DRiz 1971, 237

Schulte

Soziale Entschädigung nach dem SGB für Tumultund Gewaltschäden, Zeitschrift für Sozialreform 1974, $588 \mathrm{f}$

Sieverts, Schneider

Handwörterbuch der Kriminologie, begründet von A. Elster und H. Lingemann, 2. Aufl. BerIin, NY. 1975

Southern Californian Law Review, Vol. 43, Nr. 1 , Symposium Governmental Compensation for Victims of Violence -

(Zit.: SCIR)

v. Staudingers Kommentar zum BGB, (Staudinger), 11. Aufl. 1975

Thannheiser-Wende-Zech

Handbuch des Bundesversorgungsrechts

Walter Stutz Verlag, München

Thomas/Putzo

ZPO, Kommentar, 8. Auflage 1975

XXIV 
Verhandlungen

des 49. Deutschen Juristentages, Bd. II, Sitzungsberichte,

Beschlüsse der sozialrechtlichen Arbeitsgemeinschaft, P 126, 127; RIDP 1973 S. 35

Vorberg-van Nuis

Das Recht der Kriegsbeschädigten und Kriegshinterbliebenen

- Schriftenreihe für Unterricht und Praxis in der Kriegsopferversorgung -

Fachverlag für das Versorgungswesen

Amberger \& Maschmeyer, Herford

Weber

Schwerbehindertengesetz

Kommentar

Verlag J.P. Bachem, Köln

Weinig

Vergiftung in: Ponsold, Lehrbuch der gerichtlichen Medizin, 3. Aufi. 1967, Stuttgart S. $395 \mathrm{ff}$.

Welzel

Das Deutsche Strafrecht, 11. Auflage, Berlin 1969

Wilke-Wunderlich

Bundesversorgungsgesetz

- Handkommentar - 4. Auflage

WaIter Stutz Verlag, München

\section{Zacher}

Sozialgesetzbuch (Allgemeiner Teil)

Kommentar -Loseblattausgabe - 
Iiteraturverzeichnis

Verlag R.S. Schulz, Percha a. Starnberger See

$\underline{\text { Zipf }}$

Die Bedeutung der Viktimologie für die Strafrechtspflege, MschrKrim 1970, 1 ff.

ders.,

Kriminalpolitik - Eine Einführung in die Grundlagen, Karlsruhe 1973 